\title{
Die Deutschen Dialekte in Sibirien Russland
}

\begin{abstract}
Gilfanova Farida
$\mathrm{PhD}$ in Philology, Associate Professor at the department of foreign languages and intercultural professional communication

Gilfanov Rawil
\end{abstract}

$\mathrm{PhD}$ in Philology, lecture at the department of foreign languages and intercultural professional communication, Tyumen State University, Tyumen, Russia

\begin{abstract}
Die Personennamen jeder Epoche, der vielfältigen Sprachen bewahren die Merkmale der Dialekte und zwar der Mundarten. Welche Sprachelemente verschwinden, was hält sich bis Gegenwart, was ist mit der Kommunikation der Russlanddeutschen untereinander sind Fragen, die uns in dieser Untersuchung interessieren. Die Verbreitung der deutschen Dialekte in Sibirien ist historisch bedingt. Die Benennung „Deutsche in Sibirien“ findet die erste Erwähnung in der westeuropäischen Literatur im Jahre 1427. Die ersten Angaben von einem Deutschen in Sibirien gehört zum Anfang des XV. Jahrhunderts. 1410 war in Sibirien bairischer Landesknecht Johann Schildberger registriert worden. Er kam als Gefangene vom Khan Jedigej, der zu jener Zeit Sibirien eroberte.Im XVI. Jahrhundert waren westliche Gebiete von Sibirien auf die Karten von Sebastian Münster und Gerhard Mercator festgehalten worden. Sebastian Münster (1489 - 1552), wie bekannt ist, war Kosmograph und Hebräist, er verfasste hebräische und aramäische Grammatiken und Wörterbücher. Gerhard Mercator (1512 - 1594) war bekannter Kartograph und Geograph und er entwickelte den ersten modernen Atlas (1585) [Feldbaum 1999]. Mit dem Beginn des Angliederprozesses von Sibirien zu Russland am Ende des XVI. Jahrhunderts wurden alle Ausländer aktiv zur Erschließungsdienst von Neugebieten angezogen. Unter innen waren Ankömmlinge aus Baltikum, Deutschland, Niederlanden, nordischen Ländern, die in Russland alle zusammen als Deutschen genannt wurden.
\end{abstract}

Keywords: Die Deutschen Dialekte in Sibirien Russland

\section{Introduction}

Viele Ausländer, meistens Deutschen, kamen nach Sibirien im XVII. Jahrhundert. Die Deutschen wurden als hochqualifizierte Fachleute und zwar als Beamten, Offiziere, Bergarbeiter, Ärzte eingeladen, die zudem höher als Ortsansässige bezahlt wurden. Nach den Angaben von M.P. Alexejew kamen in Sibirien zur genannten Zeit 52 Deutsche. 1661 wurden in der Stadt Tobolsk Dutzende von ausländischen Offiziere, Militärinstrukteure registriert und zwar: I. Egart, J. von Heiden, A. Ling, J. von Remes, U. Skieter, W.F. Falsenz, D. Fiedernissen, E. Schönbek, J. Erenk, G.A. Meller Einige von innen hatten schöne Karriere gemacht und wurden in eine höhere Dienststellung eingesetzt. Die soziale Gewichtigkeit der Träger der Dialekte gehört zu den wichtigsten Faktoren der Erhaltung der deutschen Mundarten in Russland.

Unter den Generalen des sibirischen Armeekorps waren Deutschen: I.D. Buchgolz, H. T. Kindermann, I.I. Kraft, K.F. Frauendorf, I.I. Weinmarn, I.I. Springer, G.E. Strandmann, G.I.Glasenap, K.F. Klodt von Jurgensburg [Alexejew 1941]. Diese Familiennamen sind in Russland erhalten geblieben dank dem Beschluss vom Zaren Peter dem Ersten über die Zusammenstellung der Matrikeln mit dem Ziel der Registrierung der Bevölkerung..

Was den Begriff „Familia“ betrifft, kommt das Wort aus dem Lateinischen und wurde in Europa in den Bedeutungen „Das Ehepaar (mit den Kindern), die kleinste Zelle der Gesellschaft" und "die Generation“ verbreitet. Die zweite Bedeutung des Wortes war "Beiname, die Benennung" weniger gebräuchlich. W.I. Dall bewahrt in seinem erklärenden Wörterbuch auch die ersten Bedeutungen "Das Ehepaar" und „die Generation“ [Dall 2002, B. 4]. 
A.W. Superanskaja zählt die Gattungs-, Familien-, und dynastische Namen zu den Personennamen, die eine und dasselbe Denotate haben - die Kollektive der Menschen mit den unterscheidenden Merkmalen und die älteste unter innen ist die Gentilgemeinschaft [Superanskaja 1973: 175].

In die russische Sprache kam das Wort „Familia" in der Petersepoche und im Wörterbuch von S.I. Oshegow steht an der ersten Stelle die Bedeutung „Beiname, mit dem eine Person benannt und durch männliche Linie vererbt wird“. Die zweite Bedeutung des Wortes „Familia“ ist „die Generation“. Beispielweise, die älteste Familie Jusupows war die reichste in seiner Zeit. Die letzte Bedeutung des Wortes „Familia", die in der deutschen Sprache als erste, im Sinne "Das Ehepaar" bleibt, ist im Russischen veraltet und wird heute in diesem Sinne nicht gebraucht (vgl.: Всей фамилией отправились в гости").

Und es entsteht die Frage, in welchem Verhältnis Eigenname und Bedeutung sind, das betrachten wir ganz kurz. Am deutlichsten unterscheiden sich Eigennamen und Gattungsnamen im Aspekt ihrer Bedeutung, schreibt Gerhard Koß [Koß 1995 : 458]. Der Eigenname dient dazu, auf ein singuläres Objekt (eine Person, einen Ort etc.) zu referieren. Die Extension oder der Bedeutungsumfang des Eigennamen bleibt also fest. Die Bestimmung des Begriffsinhalts (der Intension) von Eigennamen ist in der Fachdiskussion schwieriger. Einige Semantiker gehen davon aus, dass Eigennamen bedeutungsleer sind und ihre Funktion sich auf die Referenz (das Verweisen) beschränkt. Andere Semantiker postulieren das Gegenteil und subsumieren alle Fakten und Sachverhalte unter die Bedeutung des Eigennamens.

Die Vertreter der Position der Bedeutungsleere von Eigennamen können nicht erklären, dass mit einigen Eigennamen minimales Bedeutungswissen in Form von Merkmalen vermittelt wird, z.B. eine Person, die wir mit dem Namen Bernhard bezeichnen, ist männlich. Die Bedeutungsmaximalisten können nicht erklären, dass ein minimales Wissen über den Träger eines Namens ausreicht, um den Namen korrekt verwenden zu können (z. B. „Goethe war ein Schriftsteller" oder „ich habe noch nie was von Goethe gelesen").

Eine Kompromissposition zwischen diesen beiden Positionen lautet, dass das Wissen über einen Eigennamenträger von Person zu Person sehr unterschiedlich sein kann. Aber es muss einen minimalen gemeinsamen Kern haben, damit diese Personen sich über diesen Eigennamenträger sinnvoll verständigen können.

Manches Wort dient zugleich als Gattungsname und als Eigenname eines typischen Exemplars, z. B. Sonne, Gott, Salz. Dabei können wichtige Eigenschaften des typischen Exemplars das unterscheidende Merkmal der Gattung sein. Das Wort "Familia" dient als Gattungsname in der deutschen Sprache und als Eigenname in der russischen Sprache. Was die Bestimmung des Begriffsinhalts (der Intension) von Eigennamen und Gattungsnamen „Familia“ in russischer und deutscher Sprachen betrifft, ist eine Bedeutung dominierende und diese Bedeutung kann in anderer Sprache nicht gebräuchlich sein.

Am Anfang des Artikels haben wir den Begriff „Familia“ im Deutschen und Russischen geklärt. Die ersten russischen und tatarischen Familiennamen erschienen in den XV - XVI. Jahrhunderten bei den Vertretern des Höchstandes der Bevölkerung. Der Mittelstand bekam Familiennamen in der zweiten Hälfte der XVIII - XIX. Jahrhunderte. Es geschah nicht gleichzeitig in allen Orten, sondern etappenweise und es hing davon ab, wie schnell die Bevölkerungsschichten berechtigt worden waren [Gilfanowa 2008].

Der Beschluss über die Zusammenstellung der Matrikeln in Russland wurde 1718 mit dem Ziel der Registrierung der Bevölkerung vorgenommen. Und von der vierten Revision (1782) an, sind so genannte ,jasaschnye“ Tataren Sibiriens registriert worden. Matrikelangaben enthielten nur Namen der Väter und danach folgten die Namen der Kinder. Da gab es keine Frauennamen. Von der Mitte des XVIII. Jahrhunderts wurden auch die Frauennamen festgeschrieben. Und im XIX. Jahrhundert erschienen Familiennamen, die von Vatersnamen mit den Suffixen - ow/ -owa gebildet worden waren. Die altertümlichen Besonderheiten der kontaktierenden Dialekte spielen große Rolle bei der Dialektmischung in den Regionen.

Russlanddeutschen erhielten ihre Dialektvielfalt ungeachtet der Verbindung mit mehreren Kulturen, die in Russland vorhanden waren. Die Zahl und die Qualität der anfänglich vertretenen Dialekte sind wichtige Faktoren, die zur Konvergenz der deutschen und sibirischtatarischen Mundarten in einer Siedlung führen könnten.

Vom XVIII. bis XIX. Jahrhundert kommen in den Matrikeln von Sibirientataren folgende Familiennamen in russischer Schreibweise vor: von Adam (Адамов / Атамов), Albert (Альбертов / Алпертов), Bauer (Бауров / Пауров / Паеров), Harrasz (Харрасов), Hirth (Хиртов), Schmidt (Шмидов / Шемитов), Schneider (Шнайдеров / Шнейдеров), Tischmann (Тышманов / Тишманов) [Gilfanowa 2008]. Solche Familienabbildungen treffen wir nur in den Matrikeln von Sibirientataren. 
Massenumsiedlung der Deutschen nach Russland begann Mitte XVIII. Jahrhundert, wenn 2 Manifeste von Katharina der Zweiten herausgegebenen waren.

Als die Deutschen, dem Aufruf von Zarin Katharina folgend, 1764 nach Russland kamen, brachten sie eine unzahlbare Vielfalt an deutschen Dialekten ins Land mit. Es gab Dörfer an der Wolga, nach den Angaben des Sprachforschers Peter Rosenberg, in denen über 100 verschiedene Dialekte gesprochen wurden. Da siedelten Hessen neben Rheinländern, Württemberger neben Pfälzern, Elsässer und Lothringer, Niederländer und Schweizer [Rosenberg 2011].

Wir interessieren uns, welche Elemente der deutschen Dialekte und zwar Mundarten in Sibirien erhalten sind.

Als Kommunikationsmittel hat die Mundart seit dem Aufkommen der Gemeinsprache, wie aus der deutschen Sprachgeschichte bekannt ist, immer mehr an Bedeutung verloren. Seither vollzieht sich ein Prozess fortschreitender Annäherung der Mundarten an die übergeordneten Normen der Gemein- und Umgangsprache. Der Prozess der Angleichung der Mundart an die Gemeinsprache führt nicht nur über die Städte. Der direkte Einfluss von Schule und Nachrichtenmitteln jeder Art wirkt auch fern der Stadt. Mit der Aufhebung des ökonomischen und sozialen Unterschiedes von Stadt und Land wird die Mundart bald nur noch als kulturelles Erbe betrachtet werden können.

Die Sprache als wichtiges Mittel menschlicher Kommunikation entwickelt sich mit der Gesellschaft und ist in steter Bewegung und Veränderung. Sie wird immer bereichert. Wortbestand, Grammatik und Stil passen sich im Laufe der Zeit veränderten Bedingungen an und erlauben eine kontinuierliche und jederzeit angemessene Verständigung. Es gibt neben sprachexternen Einwirkungen auf die sprachliche Entwicklung auch sprachinterne Veränderungen. Diese Veränderungen in der Sprache gehen nicht explosiv, sondern evolutionär vor sich, denn die Sprache muss in jeder Entwicklungsphase der Gesellschaft als lebensnotwendiges Verständigungsmittel funktionsbereit sein. Im Allgemeinen treten bei Wandlungen im Sprachgebrauch zunächst fakultative Varianten auf, die eine Zeitlang mehr oder weniger gleichberechtigt neben den bisherigen Formen stehen. Die neue Form wird dann gleichzeitig als sprachliche Parallelnorm anerkannt.

In Folge verschiedener Migrationsprozesse der Deutschen entstanden in Russland durch Konvergenz- und Divergenzeinflüsse neue Mischmundarten der Russlanddeutschen. In solchen Fällen beginnen Misch- und Ausgleichsprozesse, z. B die deutschen Namen sind in den Matrikeln von Sibirientataren auf solche Weise festgeschrieben worden: Bauer (d.) = Пауров (sibirtat.), Бауров (tat.), Schmidt (d.) = Шмидов (tat.) / Шемитов (sibirtat.). Zu einem wichtigen Faktor der Konvergenzprozesse sollte auch die interdialektale Identifizierung gerechnet werden. Die Sibirientataren identifizieren Familiennamen Пауров, Бауров mit eigenem Dialekt, sie nehmen fremde Laute [au] an und solche Laute werden im Laufe des längeren Zusammenlebens akzeptiert. Aber die Familiennamen in der Form Bauerow, Schmidtow treffen wir bei Deutschen nicht d.h. fremde Laute eines fremden Dialekts mit [-ow, -owa] sind nicht anerkannt und von der deutschen Sprache abgelehnt.

In der Mundart des Dorfes an der Wolga wird der Diphthong ei nur in wenigen Wörtern gesprochen z.B. heilig, Geist, sonst aber spricht man anstatt dieses Lautes das lange a : Eimer - Aamer. Ein anderes Beispiel könnte der Konsonantenwechsel b durch w, wie etwa in Arbeit - Arweit, aber - awer sein. Man kann solche interdialektale Identifizierungsmerkmale nicht nur im phonologischen Beriech finden, sondern auch im Bereich der Lexik und Morphologie.

Der Prozess des Ausgleichs kann durch verschiedene Faktoren entweder gehemmt oder beschleunigt werden. Beispielweise in Sibirien, wo allein die Entfernung für sprachliche Homogenität sorgt, sind die Bedingungen für die Herausbildung einer einheitlichen Verkehrssprache viel günstiger als in den voneinander entfernt liegenden Regionen. Meistens wird die russische Sprache teilweise mit deutschen Dialekten vermischt. Das Mischen der Sprachen ist vor allem für die Älteren typisch. Sie verwenden die russische Partikel, wie „nu wot“ („na also"), „no konetschno“ („aber natürlich“) oder „wsjo“" (das wär s") ganz selbstverständlich mit. Sprachforscher Peter Rosenberg schreibt, dass bis vor 30 Jahren die Dialektsprecher viele Entlehnungen aus dem Russischen auch noch „eingedeutscht“ haben. Diese „integrative Kraft“ ist heute jedoch verschwunden - gerade die Jüngeren gehen unmittelbar zum Russischen über. Als Folge davon kann eine neue Mundart entstehen, die einige Elemente der Ausgangsdialekte aufweist, jedoch mit keiner der mitgebrachten Ausgangsmundart identisch ist. Die Faktoren der Dialektmischung und der Herausbildung einer einigermaßen einheitlichen örtlichen Verkehrssprache hat A. Dulson in seiner Arbeit „Probleme der Dialektmischung anhand der Sprache der Wolgadeutschen" dargestellt [Dulson $1941: 82$ - 96]. Zu diesen Faktoren gehören unter anderem:

der Einfluss der deutschen Standardsprache, die von gebildeten Leuten gesprochen wird;

die Zahl und die Qualität der anfänglich vertretenen Dialekte; 
die soziale Gewichtigkeit der Träger dieser Dialekte;

der größere oder geringere Einfluss der gemeinsamen Hochsprache;

der Einfluss der lokalen Umgangssprache der benachbarten Dörfer;

die altertümlichen Besonderheiten der kontaktierenden Dialekte;

die sozial-ökonomischen Verhältnisse des betreffenden Dorfes;

die soziale Differenzierung seiner Bevölkerung.

Anhand unseren Angaben können wir (vom Beginn der Verbreitung der deutschen Personennamen in Russland) mundartliche Gliederung der deutschen Namen von Anfang 18. bis 20. Jh. zusammenstellen.

Bayer. - Sander, Bene, Ignaz, Natz, Xaver (5).

Süddt. Fons, Aloys, Anton, Toni/Tony, Barthel, Bartho, Eustach, Fried, German, Gin, Luigi [d3i-], Maximilian, Mike, Meik, Ottomar, Pankraz, Azius, Quirin, Rudolf, Olf, Urban, Winfried, Xaver (24).

Westdt. Lucian/ Luzian, Pankrazius, Pantaleon, Pantaleo, Valerian (5)

Norddt Fredo, Ascan/ Askan, Mathi, Olaf, Piet, Runolf / Runo, Sixten / Sten, Thorleif / Torleif / Thorsten/ Torsten, Thorulf / Torulf, Ulf (10).

Oberdt Lois, Burk, Flori, Giese, Rupp (5).

Niederdt. Andrees/Andrus, Drees/ Dries, Ebbe, Ri(e)k, Hein, Jost/ Jos, Leven, Narziss, Pieter, Steffen, Wippold/ Wippo, Witold, Wito/ Witto, Wolter, Wolf (15).

Rhein. Arno, Arnold/Arnolt, Noll, Vit/ Vid / Wit (3).

Schweiz. Chrispin, Dani/ Danni, Jacob/ Jacup, Jero, Just, Luc, Manu, Mart/Marti/Martin, Urban, Urs, Yan/ Jann (11).

Fries. Eme/ Emme, Ulbert, Ulbe, Winold/Winolt, Winno, Wynant, Wyn (7).

Die meisten Namen gehören nach unseren Belegen zur süddeutschen Mundart (24). Von diesen kommen die hervogehobenen Namen mehrere Jahre hindurch vor: Anton, Toni/Tony, Barthel, Bartho, German, Mike, Meik, Rudolf. Sie sind bis in unsere Tage hinein erhalten geblieben. Alle anderen Namen sind nur bis zum 20. Jahrhundert festgeschrieben worden. Den zweiten Platz belegen die Namen der niederdeutschen Mundart (15). Und an der dritten Stelle sind die Namen norddeutscher mundartlicher Herkunft (10).

Die angeführten Angaben zeigen uns, dass das von Russlanddeutschen gesprochene Deutsch, stark dialektal gefärbt ist. Und die Zukunft der Deutschen in Russland liegt in der Erhaltung der Dialektvielfalt und der Verbindung mit mehreren Kulturen, die in Russland in Gegenwart vorhanden sind.

\section{Literatur}

[1] Alexejew M.P. Sibirien aus den Nachrichten der bekannten westeuropäischen Forschungsreisenden und Schriftsteller. Irkutsk, 1941. 609 S.

[2] Dulson A. Probleme der Dialektmischung anhand der Sprache der Wolgadeutschen. Nachrichten der Akademie der Wissenschaften UdSSR.. 1941 - S 82 -96.

[3] Feldbaum Matthias. Neues universal Lexikon, 1999. Compact Verlag München, $960 \mathrm{~S}$.

[4] Gerhard Koß Die Bedeutung der Eigennamen: Wortbedeutung/ Namenbedeutung. S. 458 - 463 // Ein internationales Handbuch zur Onomastik. 1.Teilband. Berlin und New York, 1995.

[5] Gilfanowa F.H. Ethnolinguistische Untersuchung der Anthroponymie von Tarsky und Barabinsky Tataren. 2008. $-145 \mathrm{~S}$.

[6] Rosenberg Peter. Die Dialektvielfalt in Russland. Rundschau, № 24, 2011.

[7] Superanskaja A.W. Gesamttheorie der Eigennamen. 1973.

[8] Tomilov N.A. Etnicheskaja istoria tjurkojazychnogo naselenia Zapadno-Sibirskoj ravniny v konze XVI - nachale XX vv. Novosibirsk, 1992. $270 \mathrm{~s}$.

[9] Walejew F.T. Sibirische Tataren. - Kasan, 1993. S.21 